\title{
Tumor neuroendocrino gástrico en femenino adulto joven
}

\author{
Neuroendocrine gastric tumor in young adult female \\ Olga Cristina Chamberlin Varela, * Sairy Jaqueline Leija Sánchez, * \\ Juan David Bocanegra Torres, ${ }^{\ddagger}$ José Aguirre Trigueros ${ }^{\ddagger}$
}

Femenino de 26 años, sin antecedentes heredofamiliares de importancia. Inició cinco años atrás con pérdida paulatina de peso, acudió a institución pública donde se le comentó que presentaba tumor abdominal que se encontraba fuera de tratamiento, en ese lapso cursó con embarazo, llegando a término, actualmente su hijo tiene dos años de edad. Continuó con baja de peso posterior al parto (10 kg desde su inicio); acude a urgencias por sentir masa abdominal en cuadrantes superior e inferior derechos, asociada con sensación de plenitud y pérdida del apetito. Sin otra sintomatología agregada se efectúan estudios de imagen, en los que se observa hepatomegalia que alcanza el nivel de cresta iliaca derecha, múltiples lesiones nodulares intrahepáticas que pierden la interface con el estómago y comprimen el riñón derecho (Figura 1). Se realiza biopsia guiada por tomografía, con resultados histopatológicos de
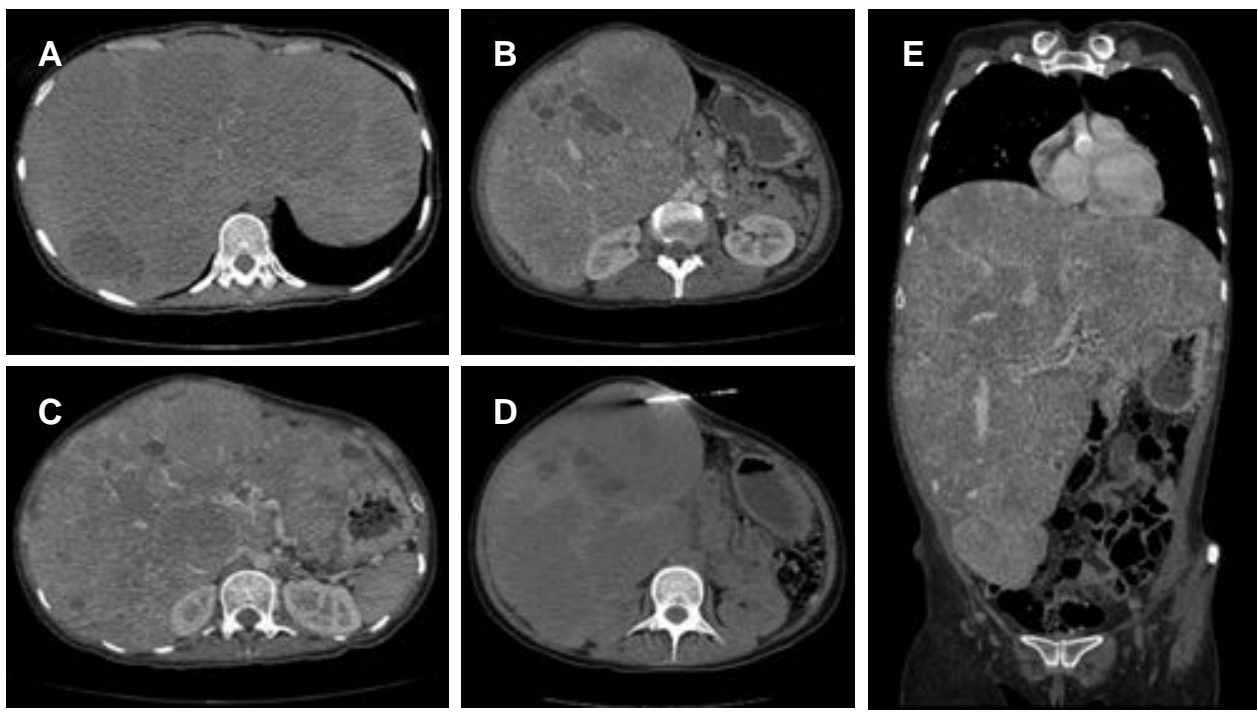

Figura 1: (A) Estudio de tomografía computarizada en fase simple en corte axial, la cual muestra hígado aumentado de tamaño, con múltiples tumoraciones hipodensas que involucran prácticamente su totalidad. (B) Tomografía computarizada contrastada en fase portal en corte axial, hígado aumentado de tamaño, lesiones hipodensas con reforzamiento periférico y compresión del riñón derecho. (C) Tomografía en corte axial en fase portal. (D) Imagen de corte axial, biopsia guiada por tomografía tipo trucut con pistola Barda de $18 \mathrm{~g}$. (E) Corte coronal que muestra la dimensión de la hepatomegalia que abarca la pelvis.

\footnotetext{
* Radiología Oncológica. Departamento de Imagenología.

₹ Patólogo. Departamento de Histopatología.
}

Hospital Ángeles León. León, Guanajuato, México.
Correspondencia:

Dra. Olga Cristina Chamberlin Varela

Correo electrónico: dra.ochamberlin@gmail.com

Aceptado: 21-06-2019.

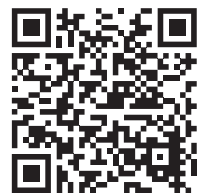



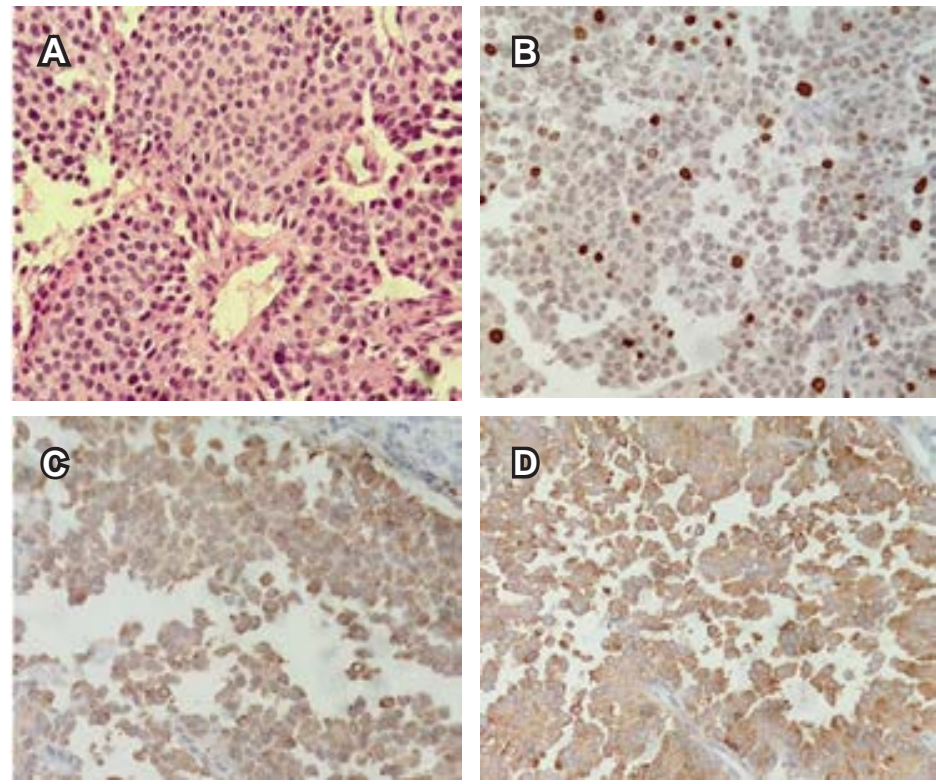

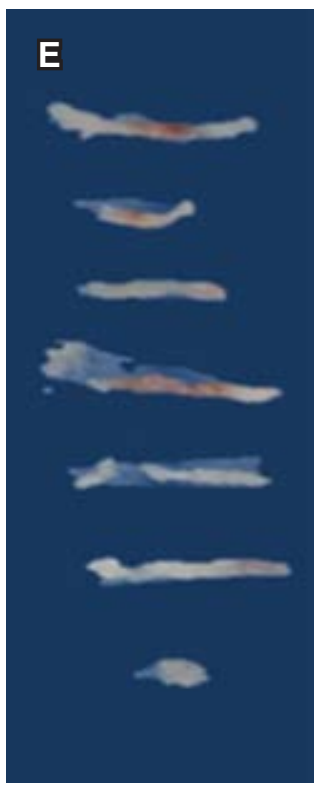

Figura 2:

(A) Células neoplásicas con núcleos redondos a ovales, cromatina en grumos gruesos "sal y pimienta", de citoplasma eosinófilo escaso y granular, mitosis atípicas de hasta cuatro por 10 campos (40x). (B) Inmunohistoquímica Ki-67 (40x). Índice de proliferación 15\%. (C) Inmunohistoquímica. Cromogranina (40x) positividad de $100 \%$ de las células neoplásicas. (D) Inmunohistoquímica. Sinaptofisina (40x), positividad de $100 \%$ de las células neoplásicas de tumor neuroendocrino bien diferenciado grado 2 de la clasificación WHO/ENETS). (E) Cilindros de biopsias obtenidas. tumor neuroendocrino gástrico (Figura 2), por lo que se deriva a oncología para valoración y manejo.

Los tumores neuroendocrinos gástricos (TNEG) son neoplasias derivadas de las células de tipo enterocromafín de la mucosa gástrica. Son lesiones raras con comportamiento indolente y diferenciación neuroendocrina, representan de 0.3 a $1.8 \%$ de todos los tipos de cáncer gástrico, ${ }^{1}$ con prevalencias de 3.2 a 1.7 por 100,000 personas en los países europeos y en los Estados Unidos, respectivamente; su incidencia ha aumentado debido al uso generalizado de endoscopia digestiva. ${ }^{2}$ Clínicamente, la mayoría de los pacientes se mantienen asintomáticos hasta que la neoplasia se encuentra en fase avanzada; los síntomas se presentan por efecto compresivo local, fibrosis o secreción de productos bioactivos, que cuando hay metástasis son secretados en sangre sin sufrir metabolismo hepático, lo cual produce una serie de síntomas conocidos como síndrome carcinoide. Los síntomas comunes a los TNEG son: dolor abdominal, pérdida de peso y cambios en el patrón intestinal. Actualmente, las técnicas de inmunohistoquímica más útiles para el diagnóstico y clasificación son la cromogranina A y la sinaptofisina. ${ }^{3}$ La supervivencia de los pacientes depende principalmente del tipo de tumor y del estadio en el momento del diagnóstico. La tasa de supervivencia a cinco años va de $7.1-21.2 \%$ de los pacientes con enfermedad metastásica a $64.5-69.1 \%$ de los pacientes sin metástasis.

\section{REFERENCIAS}

1. Basuroy R, Srirajaskanthan R, Prachalias A, Quaglia A et al. The investigation and management of gastric neuroendocrine tumours. Aliment Pharmacol Ther. 2014; 39: 1071-1084.

2. Días RA, Azevedo CB, Alban VL, Yagi KO et al. Gastric neuroendocrine tumor: review and update. Arq Bras Cir Dig. 2017; 30: 150-154.

3. Tian FX, Cai YQ, Zhuang LP, Chen MF. Clinicopathological features and prognosis of patients with gastric neuroendocrine tumors: a population-based study. Cancer Med. 2018; 7: 5359-5369. 